\title{
Use of Artificial Neural Networks in predicting rate of penetration and optimization weight on bit for several wells in Nam Rong - Doi Moi field, Vietnam
}

\author{
Hung Tien Nguyen ${ }^{1,}{ }^{*}$, Duong Hong Vu ${ }^{1}$, Vinh The Nguyen ${ }^{1}$, Doan Thi Tram ${ }^{1}$, Pham \\ Van Trung ${ }^{2}$
}

${ }^{1}$ Hanoi University of Mining and Geology, Hanoi, Vietnam

2 PVEP, Hanoi, Vietnam

\begin{abstract}
ARTICLE INFO
ABSTRACT

Article history:

Received 07th Feb. 2021

Revised $16^{\text {th }}$ May 2021

Accepted $16^{\text {th }}$ June 2021

Keywords:

Artificial Neural Network,

Nam Rong - Doi Moi field.

Optimization drilling

parameters,

Rate of Penetration,

Obtaining the maximum Rate of Penetration (ROP) by optimization of drilling parameters is the aim of every drilling engineer. This helps to save time, reduces cost and minimizes drilling problems. Since ROP depends on a lot of parameters, it is very difficult to predict it correctly. Therefore, it is necessary and important to investigate a solution for predicting ROP with high accuracy in order to determine the suitable drilling parameters. In this study, a new approach using Artificial Neural Network (ANN) has been proposed to predict ROP from real - time drilling data of several wells in Nam Rong - Doi Moi field with more than 900 datasets included important parameters such as weight on bit (WOB), weight of mud (MW), rotary speed (RPM), stand pipe pressure (SPP), flow rate (FR), torque (TQ). In the process of training the network, algorithms and the number of neurons in the hidden layer were varied to find the optimal model. The ANN model shows high accuracy when comparing to actual ROP, therefore it can be recommended as an effective and suitable method to predict ROP of other wells in research area. Besides, base on the proposed ANN model, authors carried out experiments and determine the optimal weight on bit value for the drilling interval from 1800 to $2300 \mathrm{~m}$ of wells in in Nam Rong Doi Moi field.
\end{abstract}

Copyright (C) 2021 Hanoi University of Mining and Geology. All rights reserved.

${ }^{*}$ Corresponding author

E - mail: nguyentienhung.dk@humg.edu.vn

DOI: 10.46326/JMES.2021.62(3a).05 


\title{
Ứng dụng mạng nơ-ron nhân tạo (ANN) dự báo tốc độ cơ học khoan và đề xuất giá trị tải trọng lên choòng tối ưu cho các giếng khoan dầu khí tại mỏ Nam Rồng - Đồi Mồi, Việt Nam
}

\author{
Nguyễn Tiến Hùng ${ }^{1,}$, Vũ Hồng Dương ${ }^{1}$, Nguyễn Thế Vinh ${ }^{1}$, Doãn Thị Trâm ${ }^{1}$, \\ Nguyễn Văn Trung ${ }^{2}$
}

${ }^{1}$ Khoa Dầu khí, Trường Đại học Mỏ - Địa chất, Hà Nội, Việt Nam

${ }^{2}$ Dự án block 01\&02 tại công ty PVEP, Hà Nội,Việt Nam

THÔNG TIN BÀI BÁO T TÓM TẮT

Quá trình:

Nhận bài 07/02/2021

Sưa xong 16/05/2021

Chấp nhận đăng 16/06/2021

Tù̀ khóa:

Mạng nơ-ron nhân tạo, Mỏ Nam Rồng Đồi Mồi,

Tốc độ cơ học khoan, Tối ưu hoá thông số chế độ khoan.
Nâng cao tốc độ co học khoan giúp nâng cao hiệu quả khoan, giảm thiểu nguy cơ phức tạp sự cố, thời gian thi công và giá thành phản phẩm. Tốc độ co hoc khoan phu thuộc vào nhiều tham số, gặp nhiều khó khăn trong dự báo. Vì vậy, nghiên cúu đề xuất giải pháp dự báo tốc độ co học khoan với độ chính xác cao nhằm xác định các thông số, chế độ khoan phù hợp giúp nâng cao tốc độ cơ hoc khoan là cần thiết và quan trong. Nghiên cứu này để xuất sử dụng mạng no-ron nhân tạo, nhằm dự báo tốc độ cơ học khoan tù̀ tài liệu khoan thực tế. Bộ số liệu bao gồm 900 mẫu thu được tù các giếng khoan tại mỏ Nam Rồng Đồi Mồi, chứa 6 thông số đầu vào là các thông số chế độ khoan: tải trọng lên choòng (WOB), trọng lượng riêng dung dịch khoan (MW), tốc độ quay choòng (RPM), áp suất tại vòi phun (SPP), luu lương dung dịch khoan (FR), mô men quay choòng (TQ). Trong quá trình huấn luyện mạng, các thuật toán và số nơ-ron trong lớp ẩn được thay đổi nhằm tìm ra mô hình tối uu. Mô hình mạng no-ron nhân tạo được lựa chọn cho kết quả dự báo có độ chính xác cao và có thể áp dụng cho các giếng khoan tại khu vực nghiên cúu. Ngoài $r a$, dựa vào mô hình dư báo xây dựng được, nghiên cúu này đã thử nghiệm tính toán và đưa ra đề xuất thông số tải trọng lên choòng tối uu cho khoảng độ sâu $1800 \div 2300$ m cho các giếng khoan khu vực mỏ Nam Rồng - Đồi Mồi.

C 2021 Trường Đại học Mỏ - Địa chất. Tất cả các quyền được bảo đảm.

\section{Mở đầu}

Mỏ Nam Rồng - Đồi Mồi thuộc Bể Cửu Long

*Tác giả liên hệ

E - mail: nguyentienhung.dk@humg.edu.vn DOI: 10.46326/JMES.2021.62(3a).05 nằm tại thềm lục địa Việt Nam, thuộc quản lý của Xí nghiệp Liên doanh Vietsovpetro. Các giếng khoan tại đây thường gặp nhiều phức tạp, sự cố liên quan đến mất ổn định thành giếng khi thi công trong địa tầng Mioxen và Oligoxen do đất đá chứa hàm lượng khoáng vật sét montmonrinolite cao ( 60\%) (Soloviev, Nguyen, 2015), giếng trong 
tình trạng thân trần, không chống ống thời gian dài. Vì vậy, việc nâng cao tốc độ khoan cơ học nhằm rút ngắn thời gian thi công, giảm thiểu nguy cơ sự cố khi thi công là cần thiết và quan trọng.

Dựa trên số liệu khoan thực tế trong khoảng chiều sâu $1800 \div 2300 \mathrm{~m}$ thuộc địa tầng Mioxen của 2 giếng 406 và 420 mỏ Nam Rồng - Đồi Mồi, nhóm tác giả tiến hành phân tích, thống kế số liệu (Bảng 1), xây dựng biểu đồ thể hiện sự thay đổi của thông số tải trọng lên choòng, tốc độ cơ học khoan theo chiều sâu thẳng đứng (Hình1).

Qua số liệu thống kê (Bảng 1) và biểu đồ biểu diễn sự thay đổi tải trọng lên choòng và tốc độ cơ học khoan theo chiều sâu của 2 giếng 406 và 420 (Hình 1), có một số nhận xét như sau:
- Tốc độ khoan cơ học thay đổi liên tục và không tuân theo quy luật;

- Tốc độ cơ học khoan mà 2 nhà thầu Baker Hughes và Slumberger đạt được cũng rất khác nhau. Trong khi nhà thầu Baker Hughes đạt tốc độ cơ học khoan trung bình 41,73 m/h tại giếng 406, thì nhà thầu Slumberger chỉ đạt $21,4 \mathrm{~m} / \mathrm{h}$ tại giếng 420;

- Thông số tải trọng lên choòng có ảnh hưởng lớn đến tốc độ cơ học khoan;

- Thông số tải trọng lên choòng mà 2 nhà thầu Baker Hughes và Slumberger sử dụng rất khác nhau. Trong khi nhà thầu Schlumbeger sử dụng tải trọng lên choòng dao động trong khoản $0,16 \div 6,87$ tấn, thì nhà thầu Baker Hughes sử dụng thông số này trong khoảng $5,51 \div 16,35$ tấn;

Bảng 1. Thống kê các thông số từ 2 giếng khoan 406 và 420 mỏ Nam Rồng - Đồi Mồi.

\begin{tabular}{|c|c|c|c|c|}
\hline \multicolumn{2}{|c|}{ Các tham số } & GK 406 - RCDM & GK 420RC & 2 giếng \\
\hline \multicolumn{2}{|c|}{ Tổng số mẫu } & 511 & 472 & 986 \\
\hline \multirow{2}{*}{$\begin{array}{l}\text { Chiều sâu thẳng } \\
\text { đứng }\end{array}$} & Bắt đầu & 1800 & 1800 & 1800 \\
\hline & Kết thúc & 2300 & 2300 & 2300 \\
\hline \multirow{4}{*}{$\mathrm{ROP}(\mathrm{m} / \mathrm{hr})$} & Giá trị nhỏ nhất & 22,74 & 10,03 & 10,03 \\
\hline & Giá trị lớn nhất & 54,85 & 38,75 & 54,85 \\
\hline & Giá trị trung bình & 41,73 & 21,4 & 31,94 \\
\hline & Độ lệch chuẩn & 8,67 & 6,87 & 12,84 \\
\hline \multirow{4}{*}{ WOB (ton) } & Giá trị nhỏ nhất & 5,51 & 0,16 & 0,16 \\
\hline & Giá trị lớn nhất & 16,35 & 5,53 & 16,35 \\
\hline & Giá trị trung bình & 10,41 & 2,1 & 6,41 \\
\hline & Độ lệch chuẩn & 2,52 & 0,78 & 4,56 \\
\hline \multirow{4}{*}{$\mathrm{RPM}(\mathrm{revs} / \mathrm{mn}$ ) } & Giá trị nhỏ nhất & 116 & 100 & 100 \\
\hline & Giá trị lớn nhất & 135 & 166 & 166 \\
\hline & Giá trị trung bình & 131 & 134 & 132,44 \\
\hline & Độ lệch chuẩn & 5,28 & 12,04 & 9,3 \\
\hline \multirow{4}{*}{ TQR(kg.m) } & Giá trị nhỏ nhất & 1582 & 189,2 & 189,2 \\
\hline & Giá trị lớn nhất & 2478 & 3215,5 & 3215,5 \\
\hline & Giá trị trung bình & 2068,75 & 2731,1 & 2387,5 \\
\hline & Độ lệch chuẩn & 180,24 & 255,47 & 397,29 \\
\hline \multirow{4}{*}{ FR $(1 / s)$} & Giá trị nhỏ nhất & 42,8 & 45,3 & 42,8 \\
\hline & Giá trị lớn nhất & 57,62 & 62,11 & 62,11 \\
\hline & Giá trị trung bình & 56,36 & 57,63 & 56,97 \\
\hline & Độ lệch chuẩn & 3,06 & 2,34 & 2,8 \\
\hline \multirow{4}{*}{ SPP (atm) } & Giá trị nhỏ nhất & 98,5 & 111,52 & 98,5 \\
\hline & Giá trị lớn nhất & 134,7 & 235,81 & 235,81 \\
\hline & Giá trị trung bình & 120,95 & 181,31 & 98,5 \\
\hline & Độ lệch chuẩn & 8,09 & 21,91 & 34,28 \\
\hline \multirow{4}{*}{ Mw (kg/l) } & Giá trị nhỏ nhất & 1,11 & 1,07 & 1,07 \\
\hline & Giá trị lớn nhất & 1,2 & 1,16 & 1,2 \\
\hline & Giá trị trung bình & 1,15 & 1,11 & 1,135 \\
\hline & Độ lệch chuẩn & 0,028 & 0,027 & 0,035 \\
\hline
\end{tabular}




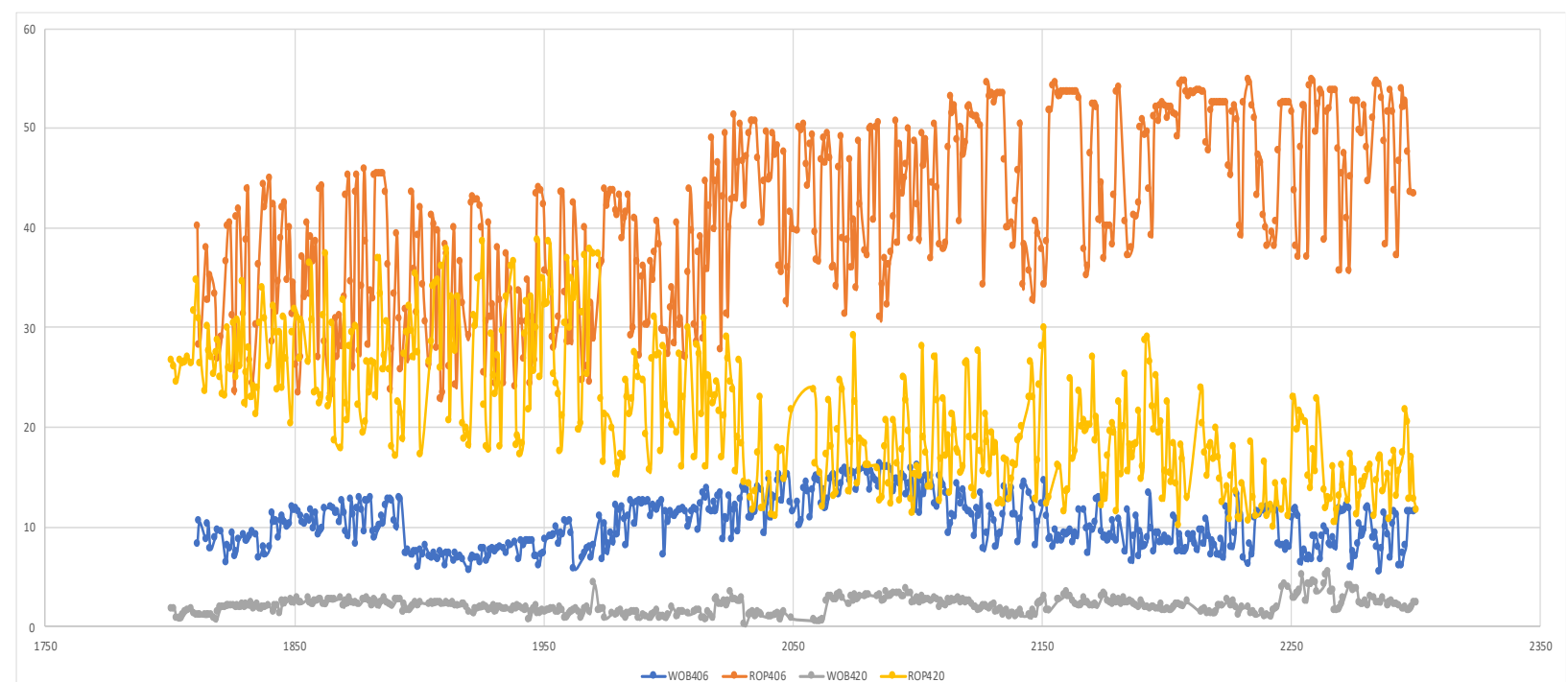

Hình 1. Sự thay đổi tải trọng lên choòng và tốc độ cơ học khoan theo chiều sâu của 2 giếng 406 và 420 mỏ Nam Rồng - Đồi Mồi, Việt Nam: WOB406 và WOB420 - Tải trọng lên choòng của giếng khoan 406 và 420; ROP406 và ROP420 - Tốc độ khoan cơ học của giếng khoan 406 và 420.

- Mặc dù nhà thầu Baker Hughes đạt tốc độ cơ học khoan cao, tuy nhiên, khoảng điều chỉnh thông số tải trọng lên choòng rất rộng $(5,51 \div 16,35$ tấn) và không tuân theo quy luật;

- Tại khoảng $1800 \div 1900$ m nhà thầu Baker Hughes sử dụng tải trọng lên choòng trung bình 10,0 tấn nhưng tốc độ cơ học khoan trung bình đạt được thấp hơn $16,6 \%$ so với khoảng $2200 \div 2300$ $m$ khi sử dụng giá trị tải trọng lên choòng nhỏ hơn (trung bình 9,1 tấn), 35,1 m/h so với 41,2 m/h.

- Khi sử dụng giá trị tải trọng lên choòng quá lớn, mặc dù vấn duy trì được tốc độ cơ học khoan ở mức cao, nhưng sẽ làm tăng chi phí năng lượng phá huỷ, giảm tuổi thọ và tiến độ choòng.

Từ những nhận xét bên trên, nhận thấy việc tìm ra phương pháp dự báo tốc độ cơ học khoan nhằm xác định giá trị tối ưu tải trọng lên choòng khi thi công trong địa tầng Mioxen mỏ Nam Rồng - Đồi Mồi nói riêng và bể Cửu Long nói chung là cần thiết và quan trọng. Việc này không những giúp tăng tốc độ cơ học khoan, đảm bảo tuổi thọ và tiến độ của choòng mà còn giảm thời gian thi công và giá thành xây dựng giếng.

Trước đây, dự báo tốc độ cơ học khoan thường sử dụng mô hình Bourgoyne và Young (1974). Hiện nay, với sự phát triển của trí tuệ nhân tạo, đã có nhiều công trình nghiên cứu chứng minh rằng mạng nơ - ron nhân tạo (ANN) là một công cụ hữu ích giúp dự báo tốc độ khoan cơ học chính xác hơn các phương pháp truyền thống
(Irawan và Tunio, 2012; Chandrasekaran và Kumar, 2020; Azim, 2020). Nghiên cứu này đã tiến hành xây dựng mô hình mạng $\mathrm{ANN}$ nhằm dự báo tốc độ cơ học khoan từ tài liệu khoan thực tế của 2 giếng khoan mỏ Nam Rồng - Đồi Mồi.

\section{Xử lý số liệu}

\subsection{Loc nhiễu}

Trong quá trình huấn luyện, các giá trị dị thường có thể coi như nhiễu vì chúng có thể ảnh hưởng tới tính chính xác và tính tổng quát của mạng ANN. Do đó, trước khi sử dụng như dữ liệu đầu vào để huấn luyện mạng, các thông số khoan được hiệu chỉnh và lọc bỏ các giá trị nhiễu dựa theo thuật toán xác định các giá trị ngoại lai Z score (Tripathy và nnk., 2013). Chỉ số Z - score được xác định bởi công thức:

$$
z=\left|X_{i}-X_{\text {mean }}\right| / S D
$$

Trong đó: $X_{\text {mean }}$ - giá trị trung bình của tham số; SD - độ lệch chuẩn của tham số

Dựa theo nghiên cứu của Tripathy và nnk. (2013), giá trị $Z$ - score được lựa chọn để xác định nhiễu trong bộ dữ liệu dựa trên tiêu chí sau:

- Khi $z<2$ giá trị phù hợp để sử dụng;

- Khi $2<z<3$ giá trị nghi ngờ, gây ảnh hưởng tới kết quả huấn luyện mạng;

- Khi $z>3$ tương ứng giá trị nhiễu cần loại bỏ. 


\subsection{Phân tích dũ liệu}

Việc lựa chọn các thông số đầu vào để huấn luyện mạng ANN là một bước quan trọng quyết định tới độ chính xác của mô hình. Để có thể đưa ra quyết định sử dụng các thông số nào làm dữ liệu đầu vào huấn luyện mạng, nhóm tác giả đã phân tích mối quan hệ giữa các thông số chế độ khoan và tốc độ cơ học khoan dựa trên bô tài liệu từ 2 giếng khoan 406 và 420 mỏ Nam Rồng - Đồi Mồi (Hình 2).

Hệ số tương quan $R^{2}$ là chỉ số thống kê đo lường mức độ mạnh yếu giữa hai biến số. Khi giá trị hệ số tương quan tiệm cận tới 1 chứng tỏ thông số đó có mối quan hệ chặt chẽ và ảnh hưởng trực tiếp đến tốc độ cơ học khoan.

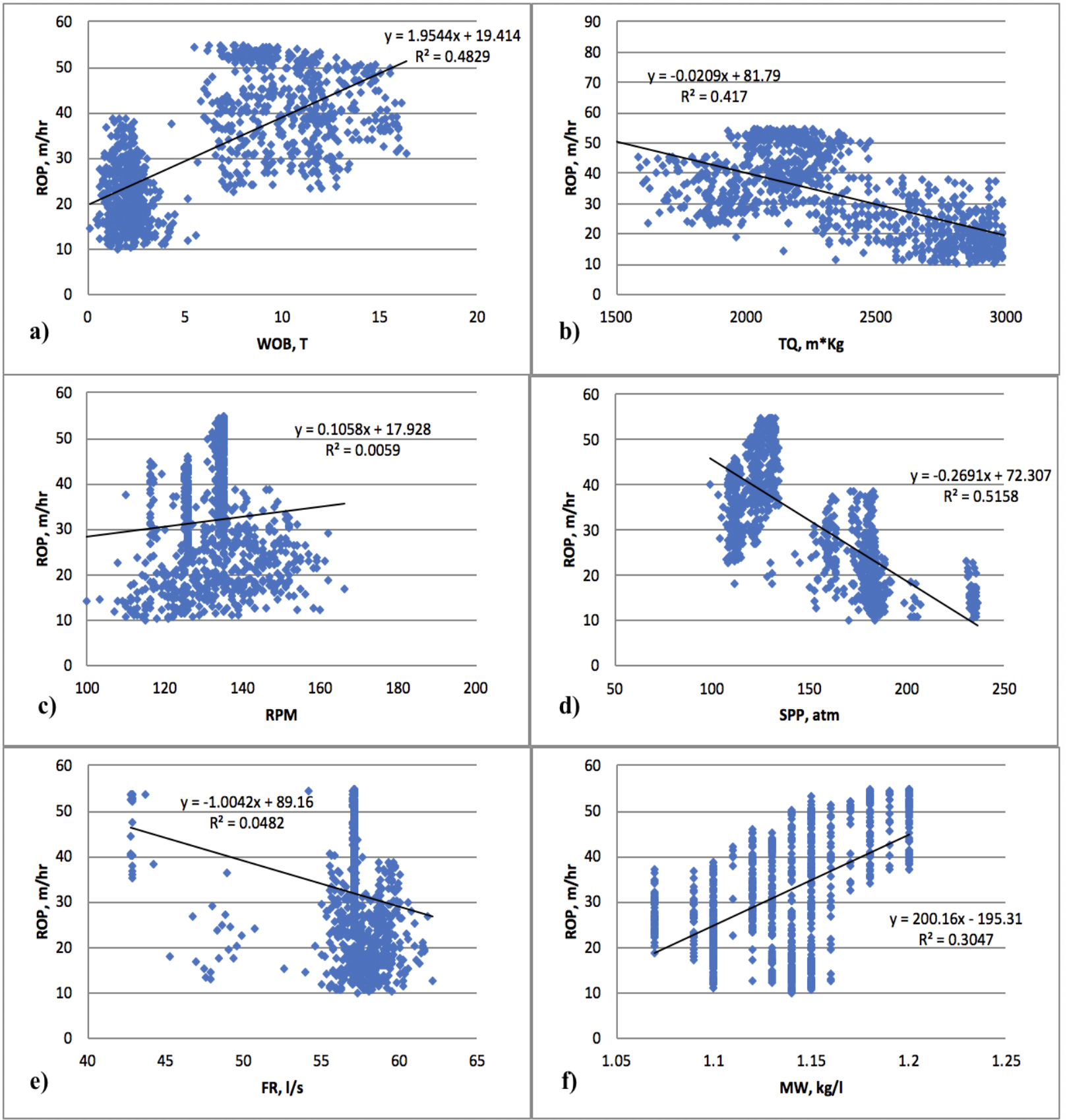

Hình 2. Hệ số tương quan giữa các thông số chế độ khoan và tốc độ cơ học khoan: a) đối với tải trọng lên choòng (WOB); b) đối với mô men quay choòng (TQ); c) đối với tốc độ quay choòng (RPM); d) đối với áp suất tại vòi phun thuỷ lực (SPP); e) đối với lư lượng dung dịch khoan (FR); f) đối với trọng lượng riêng dung dịch khoan (MW). 
Qua Hình 2 nhận thấy, các hệ số tương quan $R^{2}$ của các thông số chế độ khoan và tốc độ cơ học khoan đều nhỏ hơn 0,5 . Điều này chứng tỏ, có thể coi các thông số này như là các biến độc lập của tốc độ cơ học khoan và có thể sử dụng chúng như những thông số đầu vào để huấn luyện mạng ANN.

\subsection{Chuẩn hoá dũ liệu}

Sau khi đã lựa chọn được các thông số đầu vào, tiến hành chuẩn hoá các thông số này để đưa vào huấn luyện mạng ANN. Dữ liệu đầu vào gồm nhiều thông số và mỗi thông số lại có các đơn vị và độ lớn nhỏ khác nhau. Điều này tác động tới tính hiệu quả của thuật toán, thời gian thực hiện, quá trình hội tụ, hay thậm chí ảnh hưởng cả tới độ chính xác của mô hình ANN. Vì vậy, nhóm tác giả tiến hành điều chỉnh dữ liệu để các thông số cùng có chung một tỉ lệ (data scaling) trong khoảng $(0,1)$ bằng thuật toán Normalization dưới đây:

$$
X_{\text {normalize }}=\frac{X-X_{\min }}{X_{\max }-X_{\min }}
$$

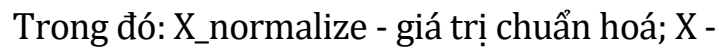
giá trị thực; X_min - giá trị cực tiểu; X_max - giá trị cực đại.

\section{Xây dựng mạng nơron nhân tạo (ANN) dự báo tốc độ cơ học khoan}

Mạng ANN là mô hình xử lý thông tin được mô phỏng dựa trên hoạt động của hệ thống thần kinh của sinh vật, bao gồm số lượng lớn các nơ ron được gắn kết để xử lý thông tin (Mohaghegh, 2000). ANN giống như bộ não con người, được học bởi kinh nghiệm (thông qua huấn luyện), có khả năng lưu giữ những kinh nghiệm hiểu biết (tri thức) và sử dụng những tri thức đó để dự đoán các dữ liệu chưa biết (unseen data). ANN được xây dựng dựa trên những giả định sau:

- Thông tin xử lý tại nhiều phần tử đơn giản, gọi là các nơ - ron;

- Tín hiệu được truyền giữa các nơ - ron thông qua các kết nối;

- Mỗi kết nối có một trọng số, thông thường được nhân với tín hiệu truyền qua;

- Mỗi nơ - ron sử dụng một hàm kích hoạt để xác định tín hiệu đầu ra theo tổng tín hiệu đầu vào.

Đặc điểm của mạng ANN có là có khả năng giải quyết được những vấn đề phức tạp bằng cách làm sáng tỏ những mối quan hệ phi tuyến, do vậy mà mạng ANN đã được ứng dụng để giải quyết các vấn đề thực tế trong nhiều lĩnh vực: công nghệ thông tin, sinh học, quản lý, kinh tế, y tế,... khi mà các quan hệ giữa các yếu tố là phi tuyến.

Nghiên cứu này đã sử dụng mạng ANN tiếp tục cung cấp (feed - forward) để dự báo tốc độ cơ học khoan dựa trên tài liệu khoan của 2 giếng khoan 406 - RCDM và 420RC mỏ Nam Rồng - Đồi Mồi. Tổng hợp các thông tin về bộ số liệu trình bày trong Bảng 1. Mạng ANN được sử dụng với thuật toán huấn luyện lan truyền ngược (back propagation) (Mohaghegh, 2000) và hàm kích hoạt tagsig để dự báo vận tốc cơ học khoan từ bộ dữ liệu mẫu trên, mạng ANN này còn được gọi là mạng nơ - ron lan truyền ngược (back propagation neural network BPNN). Bộ dữ liệu gồm 989 mẫu bao gồm các thông số: tốc độ cơ học khoan (ROP), tải trọng lên choòng (WOB), lưu lượng dung dịch khoan (FR), tốc độ quay choòng (RPM), áp suất tại vòi phun (SPP), trọng lượng riêng dung dịch khoan (MW), mô men quay choòng (TQ) (Chandrasekaran và Kumar, 2020; Azim, 2020). Bộ dữ liệu này được chia nhỏ với $70 \%$ lượng mẫu dùng để huấn luyện mạng, 15\% dùng để kiểm tra (test) và $15 \%$ để xác nhận (validation). Trong trạng thái học, tập dữ liệu mẫu đưa vào mạng ANN bao gồm cả giá trị đầu vào (inputs): tải trọng lên choòng WOB (tấn), lưu lượng dung dịch khoan FR (l/s), tốc độ quay choòng RPM (v/phút), áp suất tại vòi phun thuỷ lực SPP (atm), trọng lượng riêng dung dịch khoan MW (kg/l), mô ment tại choòng TQ (m.kg) lẫn giá trị đầu ra (output) là tốc độ cơ học khoan ROP $(\mathrm{m} / \mathrm{h})$. Các dữ liệu được tính toán và cho kết quả đầu ra. Kết quả đầu ra này của mạng ANN sau một vòng lặp (cycle hoặc iteration) được so sánh với kết quả đầu ra thực tế trong tập mẫu để rút ra sai số. Sai số này được lan truyền ngược trở lại các nơ - ron đầu ra (output neurons) và nơ - ron ẩn để các nơ - ron này điều chỉnh lại các trọng số (Hình 3).

Quá trình lan truyền theo hai chiều này được tiến hành nhiều lần, cho đến khi sai số đạt tới giá trị cực tiểu nhỏ hơn một giá trị cho phép nào đó, hoặc cho đến khi số vòng lặp đạt đến một giá trị định trước. Quá trình học của mạng ANN lan truyền ngược là quá trình học có giám sát. Trong nghiên cứu này, nhóm tác giả đã xây dựng và đánh giá các mô hình mạng ANN lan truyền ngược (BPNN) có cấu trúc chung như sau: 


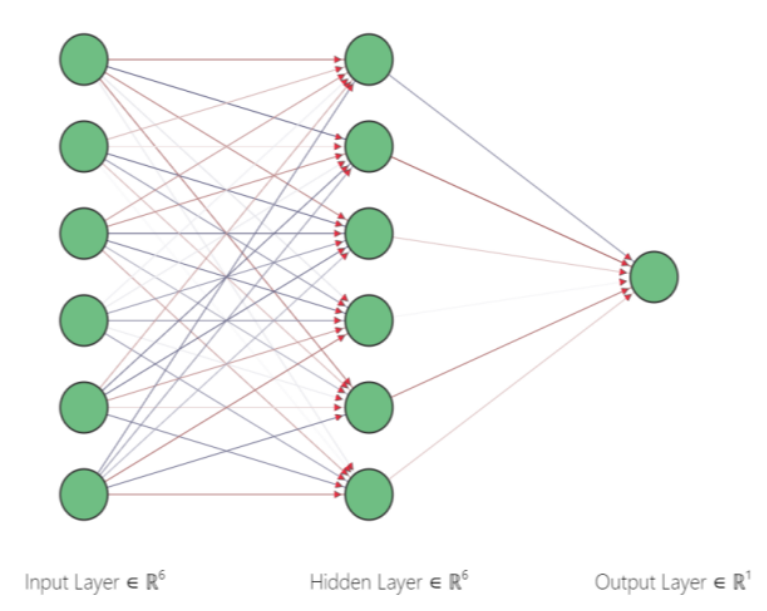

Hình 3. Mô hình biểu diễn cấu trúc mạng ANN.

Trong đó:

- Lớp đầu vào (Input layer) có 6 nơ - ron (WOB, FR, RP, SPP, MW, TQ ) và lớp đầu ra (Output layer) có 1 nơ - ron (ROP)

- Hàm kích hoạt cho lớp ẩn (Hidden layer) là tansig và hàm kích hoạt cho lớp đầu ra là pureline

Điểm khác nhau giữa các mô hình mạng là:

* Số nơ - ron ở lớp ẩn thay đổi 4\%8.

* Sử dụng 2 thuật toán học "learning" khác nhau trong toolbox của Matlab. Có nhiều thuật toán huấn luyện lan truyền ngược (backpropagation) khác nhau, chúng khác nhau về cách tính toán, dung lượng bộ nhớ và không có thuật toán nào là tốt nhất đối với mọi trường hợp (Bảng 2). Ở nghiên cứu này, với khối lượng tài liệu vừa phải, nghiên cứu này đã lựa chọn sử dụng 2 thuật toán trainlm và trainrp.

Việc lựa chọn số lượng nơ - ron tối ưu trong lớp ẩn là hết sức quan trọng, phải đảm bảo kết quả dự báo từ mạng ANN đạt được hệ số tương quan đủ tốt với dữ liệu mẫu, đồng thời số lượng nơ - ron cũng không nền sử dụng quá nhiều vì nó sẽ dẫn đến hiện tượng quá khớp (overfitting).

Từ kết quả thu được khi chạy các mô hình mạng khác nhau (các Hình 4,5), khi so sánh hệ số tương quan $R^{2}$ của cả 3 tập huấn luyện, test và validation, nghiên cứu này đã sử dụng mô hình mạng có 8 nơ - ron lớp ẩn và với thuật toán trainlm làm mô hình tối ưu để dự báo tốc độ khoan cơ học.

Từ Hình 6 có thể nhận thấy, kết quả tốc độ cơ học khoan dự báo được từ mạng ANN khi so sánh với dữ liệu thực tế có độ chính xác cao và phản ánh được xu thế thay đổi của tốc độ cơ học khoan theo chiều sâu thẳng đứng ở cả 2 giếng.

Bảng 2. Mô tả các thuật toán huấn luyện mạng ANN.

\begin{tabular}{|c|l|}
\hline Hàm & \multicolumn{1}{|c|}{ Mô tả } \\
\hline Traingd & Gradient descent cơ bản. Đáp ứng chậm, sử dụng trong chế độ incremental. \\
\hline Traingdm & Gradient descent có quán tính. Thường nhanh hơn traingd, sứ dụng trong chế độ́c. \\
\hline Traingdx & Tốc độ học thay đổi. Huấn luyện nhanh hơn traingd nhưng chỉ sứ dụng trong chế độ batch. \\
\hline Trainrp & $\begin{array}{l}\text { Resillient backpropagation. Đây là thuật toán huấn luyện chế độ batch đơn giản có hội tụ } \\
\text { nhanh và ít tốn bộ nhớ. }\end{array}$ \\
\hline Traincgf & $\begin{array}{l}\text { Thuật toán conjugate gradient fletcher reeves. Yêu cầu bộ nhớ trong các thuật toán } \\
\text { conjugate gradient. }\end{array}$ \\
\hline Traincgp & $\begin{array}{l}\text { Thuật toán conjugate polka - ribiere. Yêu cầu bộ nhớ lớn hơn traincgf ,tốc độ hội tụ nhanh } \\
\text { hơn. }\end{array}$ \\
\hline Trainscg & $\begin{array}{l}\text { Thuật toán scaled conjugate gradient. Đây là thuật toán conjugate gradient duy nhất không } \\
\text { yêu cầu định hướng tìm. }\end{array}$ \\
\hline Trainbfg & $\begin{array}{l}\text { Thuật toán quasi - newtin bfgs. Yêu cầu lưu trữ ma trận hessian và có nhiều phép tính hơn } \\
\text { các thuật toán conjugate gradient trong mỗi vòng lặp, nhưng thường hội tụ sau vài phép lặp. }\end{array}$ \\
\hline Trainoss & $\begin{array}{l}\text { Phương pháp one step secant. Đây là phương pháp kết hợp giữa phương pháp conjugate } \\
\text { gradient và phương pháp quasi - newton. }\end{array}$ \\
\hline Trainlm & $\begin{array}{l}\text { Thuật toán levengeg - marquardt. Thuật toán huấn luyện nhanh đối với mạng có kích thước } \\
\text { vưa phải,có tính chất giảm bộ nhớ khi tập huấn luyện lớn. }\end{array}$ \\
\hline Trainbr & $\begin{array}{l}\text { Thuật toán chuẩn tổng quát hóa Bayesian. Là giải thuật huấn luyện levenberg - marquardt } \\
\text { được sưa đổi tạo ra mạng tốt hơn,giảm sự phức tạp để mạng hội tụ. }\end{array}$ \\
\hline
\end{tabular}




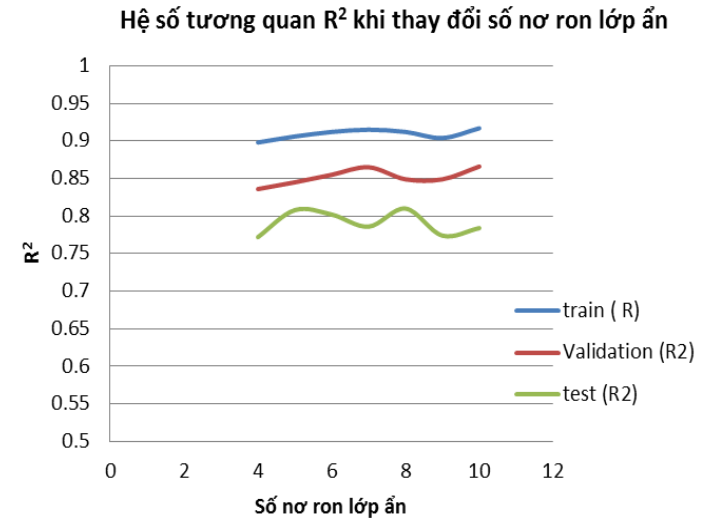

Hình 4. Kết quả dự báo khi sử dụng thuật toán trainlm.

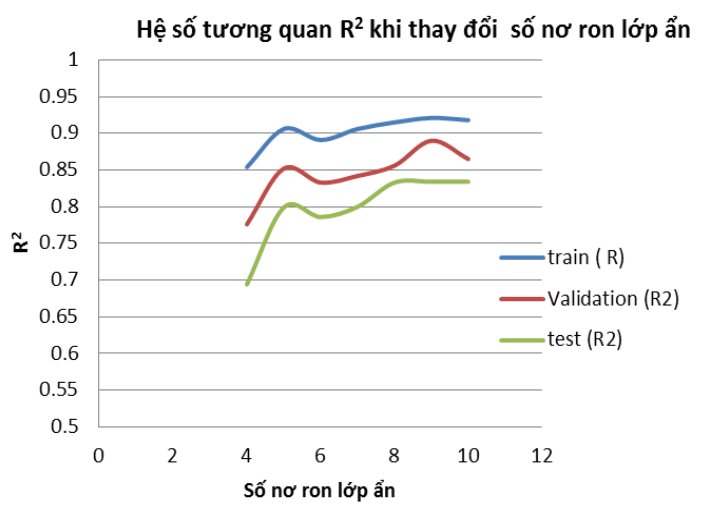

Hình 5. Kết quả dự báo khi sử dụng thuật toán trainrp.

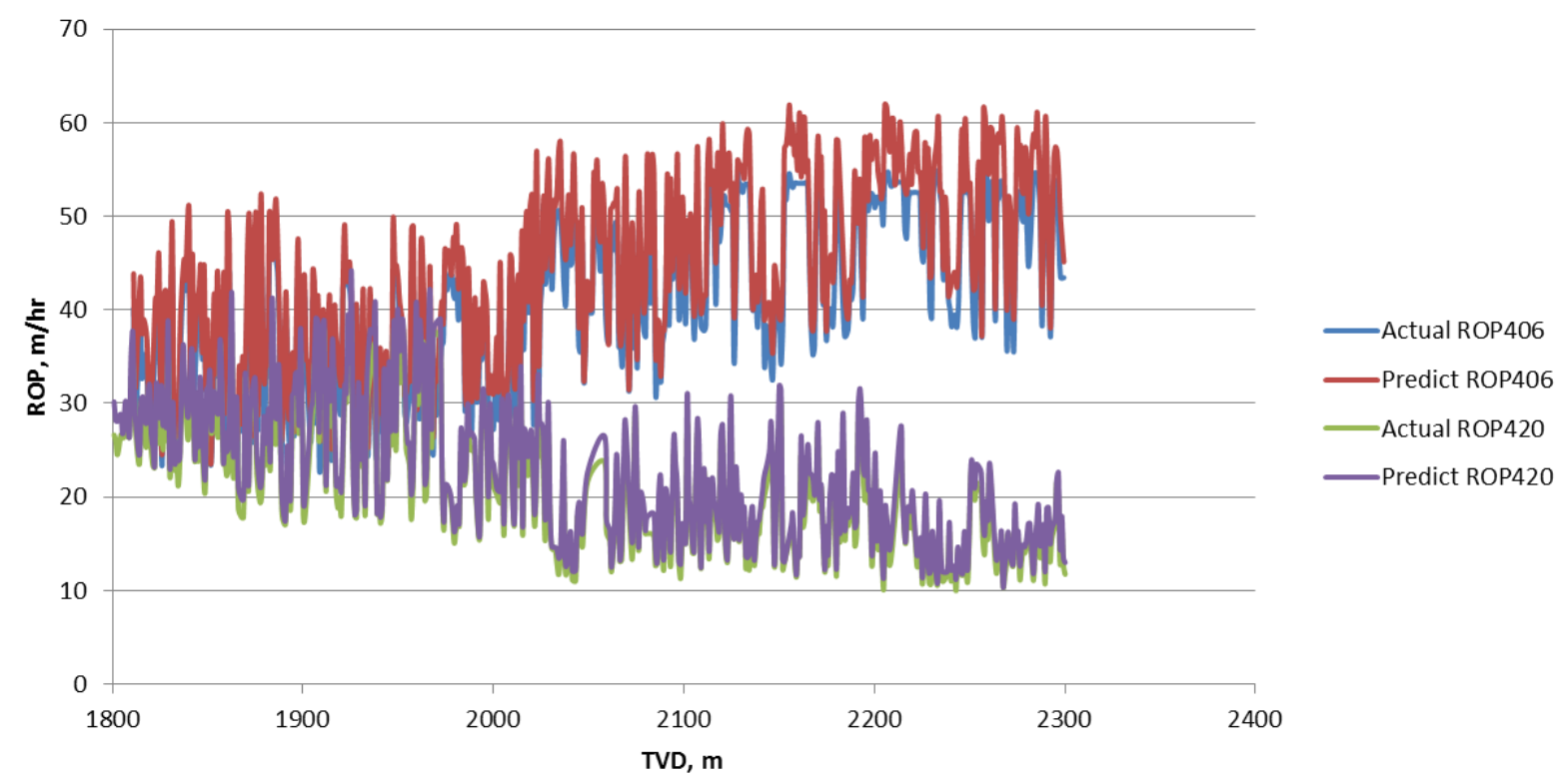

Hình 6. Tốc độ cơ học khoan dự báo tù̀ mạng ANN với dũ liệu thực tế của 2 giếng 406 và 420: Actual ROP406 và Actual ROP420 - Tốc độ co học khoan thực tế tại giếng 406 và 420; Predict ROP406 và Predict ROP420 - Tốc độ co học khoan dự báo tại giếng 406 và 420.

\section{Xác định thông số tải trọng lên choòng tối ưu nhằm nâng cao tốc độ cơ học khoan}

Để xác định thông số tải trọng lên choòng tối ưu nhằm nâng cao tốc độ cơ học khoan, nhóm tác giả tiến hành thay đổi giá trị tải trọng lên choòng lần lượt là: 2 ,4, 6, 8, 10,12, 14 tấn trong bộ dữ liệu mẫu và sử dụng mô hình ANN đã xây dựng được, nhằm dự báo vận tốc cơ học khoan đạt được ở mỗi trường hợp. Thực tế, tốc độ cơ học khoan không chỉ phụ thuộc vào thông số tải trọng lên choòng mà còn chịu ảnh hưởng của các thông số khác như: lưu lượng dung dịch khoan FR (l/s), tốc độ quay choòng RPM (v/ph), trọng lượng riêng dung dịch khoan MW, chất lượng dung dịch khoan,... Do đó, không thể đưa toàn bộ bộ dữ liệu đầu vào (input) vào mạng $A N N$ mà phải chia nhỏ bộ dữ liệu cho từng trường hợp, ví dụ: với tệp P02 đã lọc và lấy các bộ thông số đầu vào với giá trị tải trọng lên choòng dao động trong khoảng $1 \div 3$ tấn; tệp P04 là các bộ thông số đầu vào có giá trị tải trọng lên choòng nằm trong khoảng $3 \div 5$ tấn,... Kết quả là 7 bộ dữ liệu được khởi tạo P02, P04, P06, P08, P10, P12, P14 tương ứng các thông số đầu vào sử dụng tải trọng lên choòng là 2, 4, 6, 8, 10, 12, 14 tấn.

Từ 7 bộ dữ liệu trên được đưa vào mạng ANN, kết quả đầu ra (output) thu được là các giá trị dự báo vận tốc cơ học khoan. Để so sánh và lựa chọn 


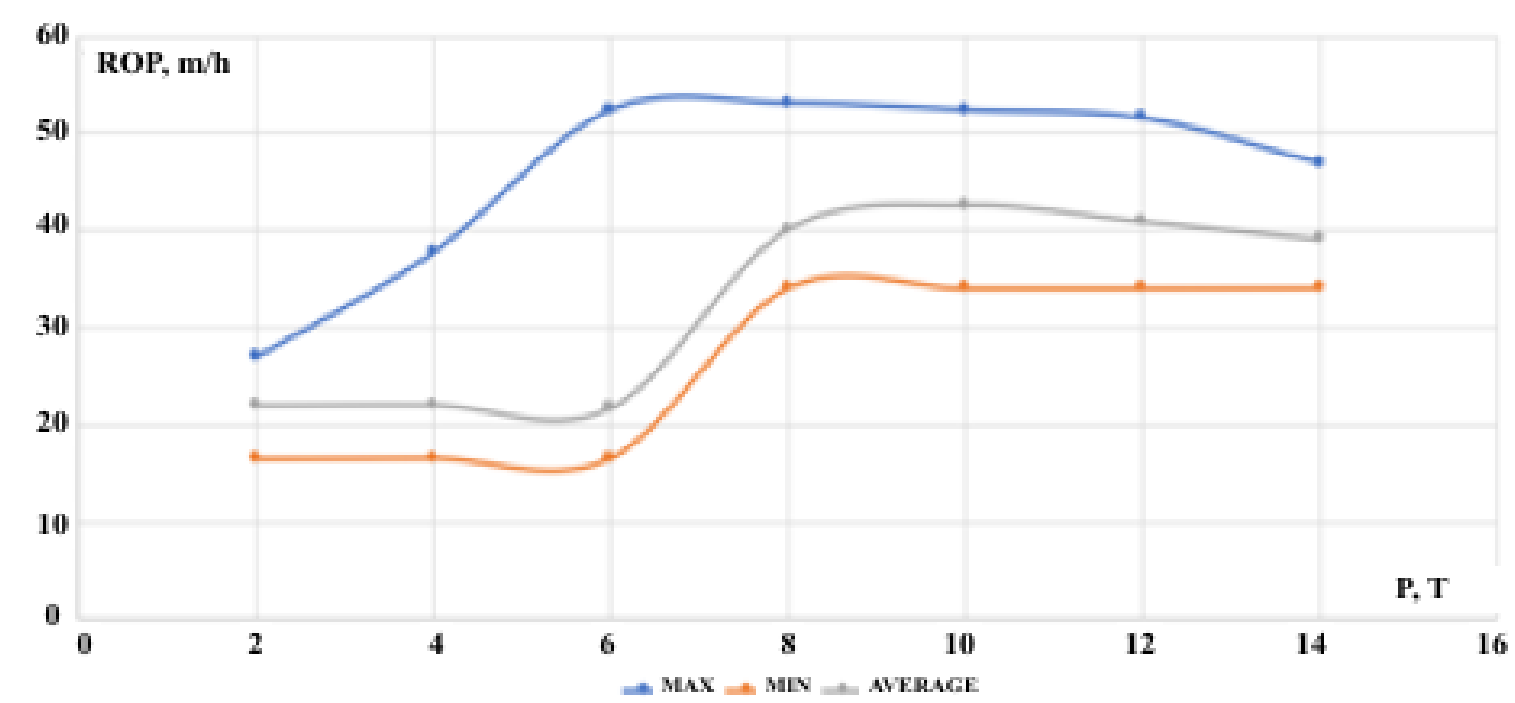

Hình 7. Biểu đồ biểu diễn sự ảnh hưởng của tải trọng lên choòng đến tốc độ cơ học khoan sau khi chạy mô hình ANN.

giá trị tải trọng lên choong tối ưu, nghiên cứu đã so sánh giá trị tốc độ cơ học khoan lớn nhất (max), nhỏ nhất (min) và trung bình trong mỗi trường hợp (Hình 7).

\section{Thảo luận và kết quả đạt được}

Qua biểu đồ thể hiện sự ảnh hưởng của thông số tải trọng lên choòng đến tốc độ cơ học khoan sau khi tiến hành chạy qua mô hình ANN (Hình 6), có một số nhận xét như sau:

- Khi tăng tải trọng lên choòng trong khoảng $2 \div 8$ tấn, tốc độ cơ học khoan tăng mạnh. Điều này phù hợp với các nghiên cứu trước đó và cho thấy khi độ ngập răng choòng tăng và mức độ làm sạch đáy giếng tốt thì tốc độ cơ học khoan sẽ tăng tuyến tính với tải trọng lên choòng (Neskoromnux, 2015; Neskoromnux, 2017; Baron và nnk., 1966);

- Khi tăng tải trọng lên choòng trong khoảng $8 \div 10$ tấn, tốc độ cơ học khoan tăng nhẹ và có xu hướng ổn định. Điều này chứng tỏ đây là khoảng giá trị hợp lý khi thi công khoan tại đây;

- Khi tăng tải trọng lên choòng trong khoảng $10 \div 14$ tấn, tốc độ cơ học khoan không tăng và có xu hướng giảm nhẹ. Điều này cũng phù hợp với các nghiên cứu trước đó khi độ ngập răng choòng tăng nhưng không đáp ứng được mức độ làm sạch đáy giếng tốt (Neskoromnux, 2015; 2017; Baron và nnk., 1966);

- Khi tải trọng lên choòng trong khoảng $10 \div 14$ tấn, không những không tăng tốc độ cơ học khoan mà còn làm tăng tiêu hao năng lượng phá huỷ, làm giảm tuổi thọ và tiến độ của choòng.

Nghiên cứu cũng nhận thấy một số tồn tại và đề xuất các giải pháp như sau:

- Thực tế khoan cho thấy, tính chất cơ lý của đất đá thay đổi rất mạnh theo chiều sâu nghiên cứu. Để đưa ra được mô hình dự báo tốc độc cơ học khoan chính xác cần chia thành các khoảng khoan chi tiết tương ứng với từng lớp đất đá. Tuy nhiên, trong nghiên cứu này do bộ dữ liệu thu thập được chỉ bao gồm tài liệu khoan thực tế của 2 giếng khoan với số lượng mẫu hạn chế nên nghiên cứu này không thể chia thành các khoảng khoan chi tiết. Để nâng cao tính chính xác và logic, trong những nghiên cứu tiếp theo sẽ bổ sung thêm tài liệu các giếng khoan lân cận.

- Khi sử dụng tài liệu của các giếng khoan khác nhau để xây dựng mô hình dự báo, hai thông số góc nghiên và góc phương vị sẽ có ảnh hưởng lên kết quả và cần được coi như 2 thông số đầu vào để huấn luyện. Tuy nhiên, bộ dữ liệu gốc thu thập được lại thiếu 2 thông số này. Vì vậy, để nâng cao tính chính xác và logic trong dự báo, nghiên cứu đề xuất bổ sung 2 thông số góc nghiêng và góc phương vị trong các nghiên cứu tiếp theo.

\section{Kết luận}

Qua các nghiên cứu bên trên, nhóm tác giả đưa ra một số kết luận như sau:

Mạng nơron nhân tạo ANN có khả năng dự 
báo tốc độ cơ học khoan tốt với mức độ chính xác cao khi tốc độ cơ học khoan dự báo gần như trùng khớp với kết quả khoan thực tế.

Mạng nơron nhân tạo ANN với thuật toán trainlm có 8 nơ - ron trong lớp ẩn cho kết quả dự báo ưu việt hơn so với các mô hình khác đối với các tệp dữ liệu này;

Để tăng độ chính xác trong dự báo của mạng ANN, cần bổ sung và cập nhật dữ liệu huấn luyện từ các giếng khoan mới;

Tải trọng lên choòng tối ưu nhằm tăng tốc độ cơ học khoan nằm trong khoản $8 \div 10$ tấn. Điều này phù hợp với kết quả khoan thực tế khi nhà thầu Baker Hughes khoan với tải trọng lên choòng trung bình trong khoảng $7 \div 13$ tấn và đạt tốc độ cơ học khoan trung bình $41,7 \mathrm{~m} / \mathrm{h}$, trong khi nhà thầu Slumberger sử dụng tải trọng lên choòng trung bình $1 \div 3$ tấn và đạt tốc độ cơ học khoan trung bình 21,4 m/h (Hình 1 ) và (Bảng 1);

Khi tăng tải trọng lên choòng từ $10 \div 14$ tấn, mặc dù có thể duy trì tốc độ cơ học khoan ở mức cao nhưng lại làm tăng mô ment quay choòng, tăng chi phí năng lượng phá huỷ, giảm tuổi thọ và tiến độ choòng. Vì vậy, đối với địa tầng Mioxen không nên dùng tải trọng lên choòng lớn như vậy;

Ứng dụng mạng ANN không những có thể tối ưu hoá thông số tải trọng lên choòng mà còn có thể tối ưu hoá các thông số chế độ khoan khác như: tốc độ quay choòng RPM, lưu lượng dung dịch khoan $\mathrm{FR}, \ldots$;

Để nâng cao hiệu quả khoan, cần tiếp tục áp dụng phương pháp sử dụng mạng ANN nhằm tối ưu hoá các thông số chế độ khoan khác như: tốc độ quay choòng RPM (v/ph), lưu lượng dung dịch khoan FR (l/s), trọng lượng riêng dung dịch khoan $\mathrm{MW}\left(\mathrm{G} / \mathrm{cm}^{3}\right), \ldots$;

Để ứng dụng rộng rãi phương pháp sử dụng mạng ANN tối ưu hoá các thông số chế độ khoan nhằm nâng cao hiệu quả khoan cho các địa tầng khác, cần bổ sung các thông số biểu thị tính chất cơ lý của đất đá theo chiều sâu trong bộ thông số đầu vào;

Nên sử dụng kết quả của nghiên cứu này như một phương pháp dự báo tốc độ cơ học khoan và tối ưu hoá thông số chế độ khoan cho các giếng khoan dầu khí tại mỏ Nam Rồng - Đồi Mồi, bể Cưu Long, Việt Nam.

\section{Đóng góp của các tác giả}

Tác giả Nguyễn Tiến Hùng lên kế hoạch, tiến hành thu thập, xử lý số liệu và kiểm tra tiến độ công việc. Nguyễn Thế Vinh và Vũ Hồng Dương thiết kế mô hình mạng nơ - ron nhân tạo (ANN) và đảm nhiệm huấn luyện mạng. Doãn Thị Trâm và Phạm Văn Trung thu thập số liệu và chỉnh sửa nội dung.

\section{Tài liệu tham khảo}

Azim Reda Abdel, (2020). Application of artificial neural network in optimizing the drilling rate of penetration of western desert Egyptian wells. Springer Nature Switzerland AG.

Baron L.I., Beron A.I., Alekhova Z. N. và nnk. (1996). Nguyên lý phá huỷ đất đá trong khoan. Khoa Học. Matx-Co'-Va, 244 trang. Барон Л. И., Берон А. И., Алехова З. Н. и другие (1966). Разрушение горных пород механическими способами при бурении скважин. Наука. М. $244 \mathrm{c}$.

Bourgoyne Jr A. T., \& Young Jr F. S. (1974). A multiple regression approach to optimal drilling and abnormal pressure detection. Society of Petroleum Engineers Journal, 14(04), 371 - 384.

Chandrasekaran Sridharan, Kumar G. Suresh. (2020). Drilling Efficiency Improvement and Rate of Penetration Optimization by Machine Learning and Data Analytics. International Journal of Mathematical, Engineering and Management Sciences, 5(3), 381 - 394.

Irawan Sonny, Tunio Saleem Qadir. (2012) Optimization of Weight on Bit During Drilling Operation Based on Rate of Penetration Model. Research Journal of Applied Sciences, Engineering and Technology, 4(12).

Neskoromnux V.V. (2015). Nguyên lý phá huỷ đất đá trong khoan thăm dò. Trường ĐH Serbria. Krasnodar. 396 trang. Нескромных В. В., (2015). Разрушение горных пород при проведении геолого - разведочных работ. Сибирский федеральный университет. Красноярск, 396 с.

Neskoromnux V.V., (2017). Nguyên lý phá huỷ đất đá trong khoan thăm dò. Trường ĐH Serbria. Krasnodar. 336 trang. Нескромных В. В., (2017). Разрушение горных пород при бурении скважин. Сибирский 
федеральный университет. Красноярск, $336 \mathrm{c}$.

Mohaghegh Shahab. (2000). Part 1 - Artificial Neural Networks, Virtual - Intelligence Applications in Petroleum Engineering. Journal of Petroleum Technology, 52(9), 64 - 73.

Soloviev N.V., Nguyen Tien Hung. (2015). Công nghệ khoan tại các mỏ dầu khí thuộc Xí nghiệp Liên doanh Việt - Nga. Tạp chí KHKT "Kỹ su Dầu khí", 2, 45-49. Соловьев Н. В., Нгуен Тиен Хунг, (2015). Разработка элементов

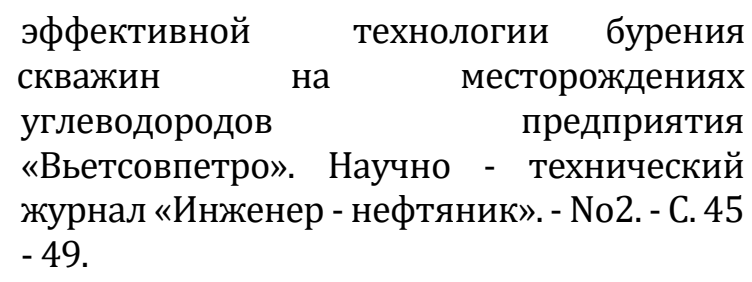

Tripathy S. S., Saxena R. K., Gupta P. K. (2013). Comparison of statistical methods for outlier detection in proficiency testing data on analysis of lead in aqueous solution. American Journal of Theoretical and Applied Statistics 2(6). 